\title{
Ein Plädoyer für kostengünstigere Labortechnologie in Entwicklungsländern
}

\section{Charlotte Eberle*}

* Keine Interessenverbindungen

Charlotte Eberle

Clinical laboratory scientist (CLS)

Schneckenmannstrasse 34

CH-8044 Zürich,

Tel: +41(0)44262 1825

charlotte.eberle@freesurf.ch
Wenn heute ein Baby in einem Entwicklungsland oder in einem von Krieg und Verfolgung zerstörten Land das Licht der Welt erblickt, so hat es eine Lebenserwartung von 33 Jahren. Hat es das Glück, in der Schweiz geboren zu werden, sind es 79 Jahre. In manchen armen unterentwickelten Ländern sterben zwei von zehn Kindern bis zum fünften Lebensjahr.

Es ist daher kein Zufall, dass Verbesserung der Gesundheit und die Verringerung der Kindersterblichkeit zuoberst auf der Liste der UNOMillenniumsziele der Völkergemeinschaften aufgeführt werden. Schaut man sich den mehrseitigen Aktionsplan betreffend das Gesundheitswesen an, wird ersichtlich, als wie wichtig der Kampf gegen HIV/Aids, Malaria und Tuberkulose eingestuft wird.

Manche Entwicklungshelfer, Ökonomen und Beamte haben Probleme mit sogenannter «Spitzenmedizin» in Entwicklungsländern. Manche Verantwortliche meinen, nur Präventivmassnahmen seien sinnvoll. Weit gefehlt! Nicht nur Vorbeugung, auch die Behandlung der anstehenden Probleme ist ein wichtiger Beitrag zur Verbesserung der Gesundheit. Eine erfolgreiche Behandlung ist möglich, wenn die Diagnose einer Krankheit sicher erhoben werden kann. Unter anderem ist dies erreichbar mit richtiger, rascher labormedizinischer Diagnosetechnik. Eine schnell gestellte, genaue klinische und labortechnische Diagnose erleichtert nicht nur die Entscheidungsfindung des Arztes, sie hilft auch die Kosten zu senken, da gezielt das richtige Medikament eingesetzt werden kann, ohne eine Vielzahl teurer Arzneimittel auszuprobieren. Gleichzeitig können allgemeine Folgeschäden durch falsch verabreichte Medikamente und Resistenzbildungen vermieden werden. Bei der Zunahme von komplizierten, kombinierten Krankheitsbildern wie HIV/Aids, Malaria und Tuberkulose ist es unabdingbar für den Arzt, diese frühzeitig einzuschätzen, bevor Schaden entstanden ist.

Dies ist nur mit Hilfe neuer, einfacher, kostengünstiger Labortechnologie möglich. Angesichts der modernen, hochtechnisierten Medizin in

\section{Résumé}

De nombreux coopérants, économistes et fonctionnaires ne sont pas favorables à l'instauration d'une médecine de pointe dans les pays en voie de développement car ils considèrent que seules les mesures de prévention sont efficaces. Le traitement des problèmes existants représente néanmoins une contribution importante pour la promotion de la santé des individus et des groupes de population. Dans de nombreux cas, il est possible de traiter une maladie avec succès si le diagnostic a pu être posé correctement. Cela présuppose une technique de diagnostic de laboratoire fiable et rapide. Celle-ci facilite non seulement la prise de décision du médecin mais permet aussi, par la prescription du médicament approprié, d'abaisser les coûts de traitement de maladies complexes telles que le sida, la malaria et la tuberculose. La rédactrice de cet article présente les nouveaux développements en matière de techniques de laboratoire, lesquelles permettent de poser rapidement et fiablement un diagnostic pour les affections comme le sida et la malaria tout en restant financièrement accessibles pour les pays pauvres.

den Wohlstandsländern ist es den armen Ländern nicht zu verübeln, dass sie auch von unserem Fortschritt profitieren möchten. Weder Barfuss- noch Luxusmedizin ist gefragt, sondern ein Mittelweg dazwischen. Es muss nicht ein «Rolls-Royce-Labor» sein, auch mit einem «Trabant» können genaue Laborresultate erhoben werden.

So weit, so gut, aber wer bezahlt die «teuren» Anschaffungen? Die Regierungen in den Entwicklungsländern sind meist überfordert damit. Dem öffentlichen Gesundheitswesen stehen in 
den armen Ländern pro Einwohner acht Franken im Jahre zur Verfügung. Drei davon stammen aus der internationalen Hilfe. Zum Vergleich: In der Schweiz betragen die Kosten für das Gesundheitswesen über 6000 Franken pro Kopf und Jahr. Es ist klar, dass es ohne erhebliche Donatoren-Hilfe keine Verbesserung in der Gesundheit geben wird. Deshalb sind die Geldgeber aus den reichen Ländern gefordert. Die meisten Spendengelder werden für Behandlung, Medikamente und medizinisches Personal verwendet. Leider fliesst wenig bis gar nichts eines Spitalbudgets in ein technisches Laborprojekt, noch weniger wird aufgewendet zur Förderung von einfachen, genauen, raschen und kostengünstigeren Technologien.

Es gibt aber erfreuliche Entwicklungen in dieser Richtung, von denen ich mich selbst überzeugen konnte.

\section{HIV/Aids:}

Die Messung der sogenannten CD4+-Zellen ist heute einer der wichtigsten Parameter bei der Behandlung von HIV/Aids-infizierten Patienten. Die CD4+-Zellen sind eine Untergruppe der weissen Blutkörperchen (Leukozyten). Sie sind für die körpereigene Abwehr zuständig und werden durch die HIV-Viren systematisch zerstört. Dies führt zu einer dramatischen Schwächung des Immunsystems und macht den Körper für zahlreiche Infektionen anfällig.

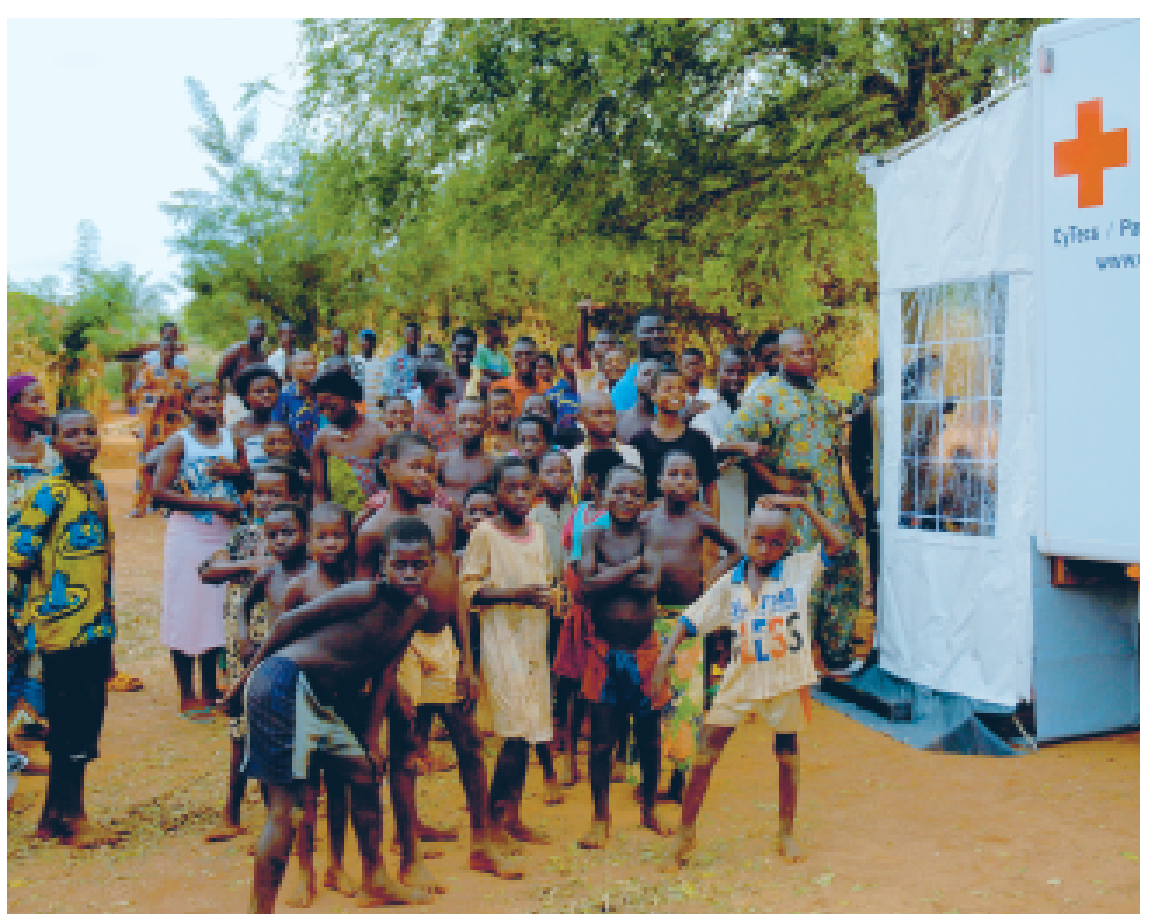

Dorfbewohner im Norden Benins vor einem mobilen Labor für kostengünstige Malaria- und HIV-Diagnostik.
Durch die Bestimmung der CD4+-Zahl erhält der betreuende Arzt entscheidende Informationen über den Verlauf einer HIV-Infektion. Er kann anhand der CD4+-Zellzahl entscheiden, wann er mit der Therapie mit antiretroviralen Medikamenten beginnen muss. Ausserdem ist eine laufende Kontrolle des Therapieerfolges möglich. Die Messung der CD4+-Zellen wird mit Durchflusszytometern durchgeführt. Problematisch ist die CD4+-Zellmessung bei Kindern. Grund ist, dass die Zahl der CD4+-Zellen bei Kindern unter 6 Jahren immer deutlich höher liegt als bei Erwachsenen. Dadurch ist eine signifikante Abnahme dieser Zellen im Falle einer HIV-Infektion schwer zu erkennen. Deshalb ist bei Kindern die Bestimmung der prozentualen CD4+-Zahl notwendig. Im Gegensatz zur Bestimmung der Gesamtzahl der CD4+-Zellen wird hier der prozentuale Anteil der CD4+-Zellen unter allen Lymphozyten ermittelt. Bislang war diese Bestimmung in den Entwicklungsländern unerschwinglich.

Die herkömmlichen Geräte können mehr als 100000 Euro kosten und jeder einzelne Test nochmals rund 100 Euro. In Anbetracht der Tatsache, dass weltweit etwa 2,2 Millionen Kinder infiziert sind, ist dies eine Katastrophe.

Seit kurzem ist ein Gerät auf dem Markt (Partec; Cyflow SL_3), das die Bestimmung des prozentualen CD4+-Wertes sowie anderer Werte (Gesamtlymphozyten, Gesamtleukozyten) in einem Arbeitsschritt möglich und auch für die armen Länder erschwinglich macht. Der kompakte («desk-top») Flowcytometer kann auch mit Batterien betrieben werden (z.B. Autobatterie) und eignet sich hervorragend für den mobilen Einsatz. Sein Anwendungsbereich umfasst die Bestimmung der Konzentration aller CD4+-TLymphozyten, der Konzentration aller Leukozyten, der Konzentration aller Lymphozyten sowie des prozentualen Anteils der CD4+-T-Lymphozyten (bezogen auf die gesamten Lymphozyten). Inklusive Reagenzien für 200 Bestimmungen beläuft sich der Preis für das Gerät auf 20850 Euro, was einem Betrag von 1.75 Euro für einen CD4-Test bzw. 2.50 Euro für einen CD4\%-Test entspricht. Dazu kommen Kosten von 1950 Euro für Installation und Schulung.

\section{Malaria:}

Laut den neuesten Daten der WHO werden zurzeit ungefähr 300 Millionen Menschen pro Jahr mit Malaria infiziert, darunter ein Kind pro Sekunde. Die Krankheit ist vermeidbar und kann behandelt werden. Einfache und rasche Diagnosetechniken sind gefordert. Die Standardmethode ist die mikroskopische Untersuchung 
des dicken und dünnen Tropfens auf dem Objektträger nach vorhergehender Färbung mit Giemsa- oder Wright-Lösung. Jedoch ist diese Prozedur aufwendig und beansprucht $z u$ viel Zeit. Wie ich selbst erlebt habe während meines Arbeitsaufenthaltes in Malawi, sind die Labors mit mehr als 20 Malariakranken pro Tag überfordert. Dazu kommen noch alle nachzukontrollierenden Patienten. Meist sind die Mikroskope alt, die Qualität der Färbungen ist ungenügend und die Labortechniker sind übermüdet, was dazu führt, dass Malariainfektionen übersehen oder nicht ausreichend Medikamente verabreicht werden. Für eine optimale Behandlung ist es unabdingbar, dass die Malariadiagnose rasch erhoben werden kann.

Für eine kostengünstige, auch für Entwicklungsländer erschwingliche Malariadiagnostik sind mittlerweile ein qualitativ gutes Mikroskop (Fluoresenz-Mikroskop CyScope der Firma Partec für 990.- Euro) und ein äusserst einfach durchzuführender, schneller Test aus demselben Haus erhältlich. Der Test besteht aus fertig vorbereiteten Objektträgern mit bereits eingetrockneten - auf dem DNAFluorochrom DAPI basierenden - Reagenzien, auf denen unter UV-Lichtanregung bei einer Wellenlänge von 365 nm der Kern des Parasiten gut sichtbar fluoresziert. Die Spezifität und Sensitivität für Plasmodium Falciparum ist mit $>95 \%$ ausgezeichnet. Zur Differenzierung der Morphologie der Plasmodien sind der dicke Tropfen und erweiterte Methoden wie PCR besser geeignet, letztere ist jedoch unmöglich durchzuführen in den armen Ländern des Südens. Mit dem neuen Test ist die Malariadiagnostik sehr einfach und sehr schnell durchzuführen. Das Verfahren ist letztlich auch billiger als die aufwendige Giemsa-Färbemethode. Die Kosten für einen Test belaufen sich auf $€$ 0.50. Auch der 102 Jahre alte Giemsa-Test lässt sich auf dem Gerät durchführen.

Die beiden vorgestellten Entwicklungen können in Anbetracht ihrer Preise als wichtiger Durchbruch im Bereich der HIV/Aids- und Malariadiagnostik bezeichnet werden. Mit beiden Geräten habe ich mich als Laborwissenschaftlerin auseinandergesetzt, und ich bin überzeugt von deren Notwendigkeit im Einsatz gegen den Kampf für die Gesundheit der betroffenen Bevöl- kerung. Hoffung besteht, dass auch bald neue Labortechniken für den Kampf gegen die Tuberkulose entwickelt werden.

Für ausführliche Informationen stehen neben der Autorin (siehe Korrespondenzadresse) zur Verfügung:

- Herr Roland Göhde, Firma Partec GmbH, Otto-Hahn-Strasse 32, D-48161 Münster; r.goehde@partec.com; www.partec.com/essentialhealth/

- Herr Dirk Angemeer, action medeor, Deutsches Medikamenten-Hilfswerk, St.-TöniserStr. 21, D-47918 Tönisvorst; dirk.angemeer @medeor.org; www.medeor-order.org

\section{Literatur}

HIV-Diagnostik:

- Cassens U, Göhde W, Kuling G, et al. Simplified volumetric flow cytometry allows feasible and accurate determination of CD4 T-lymphocytes in immunodeficient patients worldwide. Antivir Ther. 2004;9:395-405.

- Imade GE, Badung B, Pam S, Agbaji O, Egah D, Sagay AS, et al. Comparison of a new, affordable flow cytometric method and the manual magnetic bead technique for CD4 T-lymphocyte counting in a northern Nigerian setting. Clin Diagn Lab Immunol. 2005 Jan;12(1):224-7.

- Fryland M, Chaillet P, Zachariah R, Barnaba A, Bonte L, Andereassen R, et al. The Partec CyFlow Counter((R)) could provide an option for CD4+ T-cell monitoring in the context of scalingup antiretroviral treatment at the district level in Malawi. Trans R Soc Trop Med Hyg. 2006 Mar 14; [Epub ahead of print]

http://www.ncbi.nlm.nih.gov/entrez/query.fcgi $\rightarrow$ $\mathrm{cmd}=$ Retrieve $\& \mathrm{db}=$ pubmed\&dopt $=$ Abstract\&list uids $=16542690 \& q u e r y \_h l=4 \&$ itool=pubmed_ docsum.

Malariadiagnostik:

- Moody A. Rapid diagnostic tests for malaria parasites. Clin Microbiol Rev. 2002 Jan;15(1): 66-78. Review.

- A new portable fluorescence microscope and reagent system for reliable and affordable Malaria testing. Clinical Laboratory International, May 2006. http://www.cli-online.com/product. asp $\rightarrow$ pro_id $=2121$

- Rapid Malaria Diagnostics. Partec Essential Healthcare. http://www.partec.com/essentialhealth/malariatesting.html 